\title{
Idempotent Dirac density matrix for ten-electron central field inhomogeneous electron liquids in terms of electron- and kinetic energy-densities
}

\author{
N.H. March ${ }^{\mathrm{a}, \mathrm{b}}$ \\ ${ }^{a}$ Department of Physics, University of Antwerp, Antwerp, Belgium, Oxford University, Oxford, England, UK \\ $\mathrm{b}$ The Abdus Salam International Centre for Theoretical Physics, Trieste, Italy \\ Received 10 May 2007; accepted 24 May 2007 \\ Available online 8 June 2007 \\ Communicated by V.M. Agranovich
}

\begin{abstract}
A differential equation for the Dirac density matrix $\gamma\left(\mathbf{r}, \mathbf{r}^{\prime}\right)$, given ground-state electron- and kinetic energy-densities, has been derived by March and Suhai for one- and two-level occupancy. For ten-electron spin-compensated spherical systems, it is shown here that $\gamma \equiv \gamma\left[\rho, t_{g}\right]$ where $\rho$ and $t_{g}$ are electron- and kinetic energy-densities. The philosophy of March and Suhai is confirmed beyond two-level filling. An important byproduct of the present approach is an explicit expression for the one-body potential of DFT in terms of the $p$-shell electron density.

(C) 2007 Elsevier B.V. All rights reserved.
\end{abstract}

Keywords: Dirac density matrix; Kinetic energy density; Ten-electron systems

In earlier work, Howard et al. [1] have written the formal Dirac density matrix $\gamma\left(\mathbf{r}, \mathbf{r}^{\prime}\right)$ for a ten-electron central field generated inhomogeneous electron liquid as

$\gamma\left(\mathbf{r}, \mathbf{r}^{\prime}\right)=\Gamma\left(r, r^{\prime}\right)-3 f(r) f\left(r^{\prime}\right)\left|\mathbf{r}-\mathbf{r}^{\prime}\right|^{2}$.

Here $\Gamma\left(r, r^{\prime}\right)$ as indicated is a function only of $|\mathbf{r}|$ and $\left|\mathbf{r}^{\prime}\right|$, as are the factors $f$ appearing also in Eq. (1). Such a form (1) was anticipated by March and Santamaria [2], who wrote the Dirac matrix $\gamma\left(\mathbf{r}, \mathbf{r}^{\prime}\right)$ for filled $K$ plus $L$ shells, but now generated by the bare Coulomb potential energy $-Z e^{2} / r$. We shall return to this example below, following a more general discussion.

A first-order non-linear differential equation satisfied by $\gamma$ was given very recently by March and Suhai [3] for general oneand two-level occupancies for molecules and clusters. However, for these limited occupancies they stressed the functional form $\gamma \equiv \gamma\left[\rho, t_{g}\right]$ where $t_{g}$ is the (positive definite) single-particle kinetic energy related to $\gamma$ by

$t_{g}(\mathbf{r})=\left.\frac{1}{2} \frac{\partial^{2}}{\partial \mathbf{r}^{\prime} \cdot \partial \mathbf{r}} \gamma\left(\mathbf{r}, \mathbf{r}^{\prime}\right)\right|_{\mathbf{r}^{\prime}=\mathbf{r}}$.

E-mail address: grilli@ictp.it.
Let us next use the definition (2) plus the form of $\gamma\left(\mathbf{r}, \mathbf{r}^{\prime}\right)$ in Eq. (1) to obtain $t_{g}(r)$ for the present ten-electron systems. To do so, let us follow Ref. [1] and write $\Gamma\left(r, r^{\prime}\right)$ more explicitly as

$$
\Gamma\left(r, r^{\prime}\right)=\Gamma_{0}\left(r, r^{\prime}\right)+3 f(r) f\left(r^{\prime}\right)\left(r^{2}+r^{\prime 2}\right)
$$

where $\Gamma_{0}\left(r, r^{\prime}\right)$ is simply the $s$-state $(\ell=0)$ density matrix. It follows from Eqs. (3) and (1) that

$\left.\Gamma\left(r, r^{\prime}\right)\right|_{\mathbf{r}^{\prime}=\mathbf{r}} \equiv \rho(r)=\rho_{0}(r)+6 r^{2} f^{2}(r)$,

where $\rho_{0}(r)$ is the $s$-wave component of the total electron density $\rho(r)$. Denoting similarly the $p$-wave $(\ell=1)$ density by $\rho_{1}(r)$, Eq. (4) immediately relates $f(r)$ to $\rho_{1}(r)$ by

$\rho_{1}(r)=6 r^{2} f^{2}(r)$.

Howard et al. [1] also demonstrate that the $1 \mathrm{~s}$ and $2 \mathrm{~s}$ wave functions, $\psi_{10}$ and $\psi_{20}$ respectively, which in turn determine the $s$-state matrix $\Gamma_{0}\left(r, r^{\prime}\right)$ in Eq. (3), are both calculable from knowledge of $\rho_{1}(r)$. Hence, it follows that, formally at least, $\Gamma\left(r, r^{\prime}\right)$ in Eqs. (1) and (3) is determined by the $p$-wave density $\rho_{1}(r)$. But $f(r)$ is also fixed by $\rho_{1}(r)$ from Eq. (5) and it 
therefore follows from Eq. (1) that

$\gamma \equiv \gamma\left[\rho_{1}(r)\right]$

To make explicit contact with the ideas of March and Suhai [3], we next require to rewrite the functional form (6) in terms of the total ground-state electron density $\rho(r)$ and the kinetic energy $t_{g}(r)$ defined in Eq. (2). Inserting Eq. (1) into Eq. (2), and then utilizing Eq. (3), it is a straightforward matter, though involving some manipulation, to confirm that $t_{g}(r)$ takes the form

$t_{g}(r)=t_{g s}(r)+9 f^{2}(r)+6 r f(r) \frac{\partial f(r)}{\partial r}+3 r^{2}\left(\frac{\partial f(r)}{\partial r}\right)^{2}$.

But as already discussed above, $t_{g S}(r)$ is determined, at least in principle, by the $p$-wave density $\rho_{1}(r)$. Hence $t_{g}(r)$ is fixed also by $\rho_{1}(r)$ since $f(r)$ and $\rho_{1}(r)$ are related by Eq. (5). But the most direct conclusion from Eq. (7) is that the kinetic energy density $t_{g p}(r)$ of the $p$-shell in the ten-electron systems under discussion is determined explicitly by $f(r)$, since

$t_{g p}(r)=t_{g}(r)-t_{g s}(r)$.

But $f(r)$ is determined from Eq. (5) solely by the $p$-state density $\rho_{1}(r)$, and this allows the explicit relation determining $t_{p}$ to be written as

$t_{p}=\frac{\rho_{1}}{r^{2}}+\frac{\rho_{1}^{\prime 2}}{8 \rho_{1}}$.

Furthermore, from Eqs. (A1.1) and (A2.1) of Ref. [1] it follows readily by subtraction that an alternative route to the kinetic energy density $t_{p}(r)$ is via the relation

$-\frac{\partial V}{\partial r}=\frac{2}{r^{2} \rho_{1}} \frac{\partial}{\partial r}\left(r^{2} t_{p}\right)-\frac{\rho_{1}^{\prime \prime \prime}}{4 \rho_{1}}-\frac{\rho_{1}^{\prime \prime}}{2 r \rho_{1}}-\frac{3 \rho_{1}^{\prime}}{2 r^{2} \rho_{1}}-\frac{2}{r^{3}}$

where $V(r)$ is the one-body potential of DFT [4]. By combining Eqs. (9) and (10) the force $-\partial V / \partial r$ in such central field systems can be expressed solely in terms of the $p$-state electron density $\rho_{1}(r)$, a result that is complementary to Eq. (6) above. This force is most compactly written in terms of the von Weizsäcker-like kinetic energy density $t_{W 1}$, defined by $(1 / 8) \rho_{1}^{\prime 2} / \rho_{1}$, as

$-\frac{\partial V}{\partial r}=\frac{2 t_{W 1}^{\prime}}{\rho_{1}}+\frac{4 t_{W 1}}{r \rho_{1}}-\frac{\rho_{1}^{\prime \prime \prime}}{4 \rho_{1}}-\frac{\rho_{1}^{\prime \prime}}{2 r \rho_{1}}+\frac{\rho_{1}^{\prime}}{2 r^{2} \rho_{1}}-\frac{2}{r^{3}}$

which is an important byproduct of the present approach. Let us return at this stage to the special case of the bare Coulomb potential energy, namely

$V(r)=-Z e^{2} / r$.

Then March and Santamaria [2] exploited the fact that the Dirac density matrix $\gamma\left(\mathbf{r}, \mathbf{r}^{\prime}\right)$ depends only on two scalar coordinates, say $x$ and $y$, defined by

$x=r+r^{\prime}+\left|\mathbf{r}-\mathbf{r}^{\prime}\right|$

and

$y=r+r^{\prime}-\left|\mathbf{r}-\mathbf{r}^{\prime}\right|$ which can be traced back to the Runge-Lenz vector [5] being an additional constant of motion for the bare Coulomb potential (12). Or one can take linear combinations of $x$ and $y$, namely, $r+r^{\prime}$ and $\left|\mathbf{r}-\mathbf{r}^{\prime}\right|$, to bring Eq. (1) into the more explicit form

$\gamma\left(\mathbf{r}, \mathbf{r}^{\prime}\right)=\rho\left(\frac{r+r^{\prime}}{2}\right)+F\left(\frac{r+r^{\prime}}{2}\right)\left|\mathbf{r}-\mathbf{r}^{\prime}\right|^{2}$.

Immediately Eq. (15) reduces to $\left.\gamma\left(\mathbf{r}, \mathbf{r}^{\prime}\right)\right|_{\mathbf{r}^{\prime}=\mathbf{r}}=\rho(r)$, the ground-state electron density, as required. Furthermore, the explicit form of $F(r)$ is [2]:

$F(r)=-\frac{1}{64 \pi}\left(\frac{Z}{a_{0}}\right)^{5} \exp \left(-\frac{Z}{a_{0}} r\right): a_{0}=\frac{\hbar^{2}}{m e^{2}}$.

But with Eq. (15) inserted into Eq. (2) we readily find for the Coulomb potential (12) that

$t_{g}(r)=\frac{\hbar^{2}}{2 m}\left[\frac{1}{4} \frac{\partial^{2} \rho(r)}{\partial r^{2}}-6 F(r)\right]$.

Hence substituting Eq. (16) for $F(r)$ into Eq. (15) we obtain an explicit form for the March-Suhai functional $\gamma \equiv \gamma\left[\rho, t_{g}\right]$ for this example as (putting $\hbar=m=1$ ):

$$
\begin{aligned}
\gamma\left(\mathbf{r}, \mathbf{r}^{\prime}\right)= & \rho\left(\frac{r+r^{\prime}}{2}\right) \\
& +\left[\frac{1}{24}\left(\frac{\partial^{2} \rho(s)}{\partial s^{2}}\right)-\frac{t_{g}(s)}{3}\right]_{s=\frac{r+r^{\prime}}{2}}\left|\mathbf{r}-\mathbf{r}^{\prime}\right|^{2} .
\end{aligned}
$$

To sum up, the main achievement of this Letter is to demonstrate, for the ten-electron central field systems under discussion, that the idempotent Dirac density matrix $\gamma\left(\mathbf{r}, \mathbf{r}^{\prime}\right)$ can be characterized by the ground-state electron density and the kinetic energy density $t_{g}(\mathbf{r})$, that is

$\gamma \equiv \gamma\left[\rho, t_{g}\right]$

Of course, the theorems of density functional theory (DFT) [4] tell us that $t_{g} \equiv t_{g}[\rho]$ but unfortunately, to date, this functional remains unknown. However, $t_{g}$ is related to the density matrix $\gamma$ via Eq. (2), and so the known formal result of DFT that $\gamma \equiv \gamma[\rho]$ is then recovered from Eq. (16) The philosophy of March and Suhai [3], based previously on one- and two-level filling and embodied in Eq. (19), is here extended to ten-electron central field systems. Neon-like atomic ions represent one area of application, plus the almost spherical molecule $\mathrm{CH}_{4}$. The class of molecules $\mathrm{GeH}_{4}, \mathrm{SiH}_{4}$ and $\mathrm{CH}_{4}$ were treated by the writer in early work [6] using the simplest form of DFT, namely the Thomas-Fermi statistical method (see also [7] and [8]). Further work on the Dirac density matrix for $\mathrm{CH}_{4}$ would seem worthwhile in the context outlined above.

\section{Acknowledgements}

The author wishes to acknowledge that this work was carried out during a visit to the Abdus Salam International Centre for Theoretical Physics, Trieste, Italy. He thanks Professor V.E. Kravtsov for his generous hospitality and for the stimulating atmosphere in which the present study was made. He also thanks Professor P.W. Ayers for valuable comments pertaining to the general area of this work. 


\section{References}

[1] I.A. Howard, N.H. March, A. Nagy, V.E. Van Doren, Phys. Chem. Liquids 40 (2002) 47.

[2] N.H. March, R. Santamaria, Phys. Rev. A 38 (1988) 5002.

[3] N.H. March, S. Suhai, Phys. Lett. A 360 (2007) 665.

[4] R.G. Parr, W. Yang, Density-Functional Theory of Atoms and Molecules, Oxford Univ. Press, New York, 1989.
[5] See, for example, E. Merzbacher, Quantum Mechanics, Wiley, New York, 1970, p. 411

[6] N.H. March, Proc. Cambridge Philos. Soc. 43 (1952) 665.

[7] D.P. Clougherty, X. Zhu, Phys. Rev. A 56 (1997) 632.

[8] F. Despa, Phys. Rev. B 57 (1998) 7335. 